\title{
Has the phasing out of stavudine in accordance with changes in WHO guidelines led to a decrease in single-drug substitutions in first-line antiretroviral therapy for HIV in sub-Saharan Africa?
}

\author{
Alana T. Brennan ${ }^{\mathrm{a}, \mathrm{b}, \mathrm{c}}$, Mary-Ann Davies ${ }^{\mathrm{d}}$, Jacob Bor ${ }^{\mathrm{a}}$, \\ Gilles Wandeler ${ }^{\text {e,f }}$, Kathryn Stinson ${ }^{\mathrm{d}}$, Robin Wood ${ }^{\mathrm{g}}$, Hans Prozesky ${ }^{\text {, }}$, \\ Frank Tanseri, Geoffrey Fatti ${ }^{i}$, Andrew Boulle ${ }^{\mathrm{d}, \mathrm{k}}$, Izukanji Sikazwe', \\ Kara Wools-Kaloustian ${ }^{\mathrm{m}}$, Constantin Yiannoutsos ${ }^{\mathrm{m}, \mathrm{n}}$, Valériane Leroy ${ }^{\mathbf{0}}$, \\ Nathalie de Rekeneire ${ }^{p}$ and Matthew P. Fox ${ }^{a, b, c}$
}

\begin{abstract}
Objective: We assessed the relationship between phasing out stavudine in first-line antiretroviral therapy (ART) in accordance with WHO 2010 policy and single-drug substitutions (SDS) (substituting the nucleoside reverse transcriptase inhibitor in firstline ART) in sub-Saharan Africa.

Design: Prospective cohort analysis (International epidemiological Databases to Evaluate AIDS-Multiregional) including ART-naive, HIV-infected patients aged at least 16 years, initiating ART between January 2005 and December 2012. Before April 2010 (July 2007 in Zambia) national guidelines called for patients to initiate stavudine-based or zidovudine-based regimen, whereas thereafter tenofovir or zidovudine replaced stavudine in first-line ART.

Methods: We evaluated the frequency of stavudine use and SDS by calendar year 2004-2014. Competing risk regression was used to assess the association between nucleoside reverse transcriptase inhibitor use and SDS in the first 24 months on ART.

Results: In all, 33441 (8.9\%; 95\% confience interval 8.7-8.9\%) SDS occurred among 377656 patients in the first 24 months on ART, close to $40 \%$ of which were amongst patients on stavudine. The decrease in SDS corresponded with the phasing out of stavudine. Competing risks regression models showed that patients on tenofovir were $20-95 \%$ less likely to require a SDS than patients on stavudine, whereas patients on zidovudine had a $75-85 \%$ decrease in the hazards of SDS when compared to stavudine.
\end{abstract}

\footnotetext{
${ }^{\mathrm{a}}$ Department of Global Health, Boston University, Boston, Massachusetts, USA, ${ }^{\mathrm{b}}$ Health Economics and Epidemiology Research Office, Department of Internal Medicine, School of Clinical Medicine, Faculty of Health Sciences, University of the

Witwatersrand, Johannesburg, South Africa, ' ${ }^{\mathrm{C}}$ Department of Epidemiology, Boston University School of Public Health, Boston, Massachusetts, USA, ${ }^{\mathrm{d}}$ Centre for Infectious Disease Epidemiology and Research, School of Public Health and Family Medicine, University of Cape Town, Cape Town, South Africa, ' Department of Infectious Diseases, Bern University Hospital, University of Bern, Institute of Social and Preventive Medicine, University of Bern, Bern, Switzerland, ${ }^{\mathrm{g}}$ The Desmond Tutu HIV Centre, Institute of Infectious Disease and Molecular Medicine, Faculty of Health Sciences, University of Cape Town, hivision of Infectious Diseases, Department of Medicine, University of Stellenbosch \& Tygerberg Academic Hospital, Cape Town, 'Africa Center for Health and Population Studies, University of Kwazulu-Natal, ${ }^{\mathrm{j}}$ Kheth'Impilo AIDS Free Living, Cape Town, ${ }^{\mathrm{k}}$ Department of Health, Provincial Government of the Western Cape, Cape Town, South Africa, 'Center for Infectious Disease Research in Zambia, Lusaka, Zambia, ${ }^{m}$ Indiana University School of Medicine, Indianapolis, ${ }^{\mathrm{n}}$ Richard M. Fairbanks School of Public Health, Indiana University, Indianapolis, Indiana, USA, ${ }^{\circ}$ INSERM U1027, Université Paul Sabatier Toulouse 3, Toulouse, and P Université Bordeaux, ISPED, Centre INSERM U1219 Epidémiologie-Biostatistique, Bordeaux, France.

Correspondence to Alana T. Brennan, Department of Global Health Boston University Crosstown Center, 3rd Floor, 801 Massachusetts Ave, Boston, MA 02118, USA.
}

Tel: +1 617414 1179; e-mail: abrennan@bu.edu

Received: 18 May 2016; revised: 22 September 2016; accepted: 5 October 2016. 
Conclusion: The decline in SDS in the first 24 months on treatment appears to be associated with phasing out stavudine for zidovudine or tenofovir in first-line ART in our study. Further efforts to decrease the cost of tenofovir and zidovudine for use in this setting is warranted to substitute all patients still receiving stavudine.

Copyright (c) 2016 Wolters Kluwer Health, Inc. All rights reserved.

AIDS 2017, 31:147-157

\section{Keywords: antiretroviral therapy, drug side effects, drug toxicities, low and middle-income countries, single-drug substitution}

\section{Introduction}

In sub-Saharan Africa, an estimated 11.4 million HIVpositive individuals were receiving antiretroviral therapy (ART) by mid-2015 [1]. In low and middle-income countries (LMICs), HIV treatment programs take a public health approach that utilizes 12 antiretrovirals in four drug classes [2,3]. Since treatment options are limited in LMICs and at least three drugs from two drug classes are typically needed for effectiveness, therapeutic options need to be maximized. One way to increase the life span of first-line ART in patients in LMICs is by decreasing rates of substituting individual drugs, typically for reasons of toxicity, within the regimen [single-drug substitutions (SDS)].

Prior to the WHO guidelines change in 2010, the most frequently used nucleoside reverse transcriptase inhibitor (NRTI) alongside lamivudine was stavudine [2,3]. Stavudine is highly effective in treating HIV [4], but is associated with severe side effects [5-7]. In 2009, as a result of stavudine's poor side-effect profile, the WHO recommended replacing stavudine with tenofovir or zidovudine for initial HIV treatment [4]. In 2010, most governments in LMICs followed suit and began to phase out stavudine and replace it with tenofovir or zidovudine, for all new ART initiates [8]. Since there are fewer side effects and drug toxicities associated with tenofovir and zidovudine than stavudine [4,9-11], the switch was expected to be accompanied by a marked decrease in SDS in first-line ART.

To date, several observational studies set in LMICs have compared rates of SDS amongst patients on stavudine, tenofovir, and zidovudine-based regimens. All studies found that patients on tenofovir had about an $82 \%$ decrease in the risk of SDS [summary risk ratio 0.18, 95\% confidence interval (CI) 0.15-0.20] compared to patients on stavudine [12-20], whereas patients on zidovudine, although at higher risk of SDS compared to tenofovir, remained at lower risk of substitution compared to patients on stavudine (summary risk ratio $0.41,95 \% \mathrm{CI}$ $0.37-0.45)$ [12-17].

Whereas previous observational studies do suggest tenofovir and zidovudine are associated with fewer
SDS compared to stavudine [12-20], many had important limitations. Two studies [12,13] included patient populations that initiated tenofovir prior to implementation of the 2010 WHO policy change. These patients would be more likely to have initiated tenofovir because of contraindications to stavudine or zidovudine, leading to strong confounding by indication. We sought to use one of the largest HIV database in the world to assess whether or not the phasing out of stavudine in firstline ART in accordance with WHO 2010 policy decreased SDS in sub-Saharan Africa. This transition allows evaluation of the impact of a major policy change while accounting for secular trends in improvements in HIV treatment.

\section{Methods}

\section{Cohort description}

The International epidemiological Databases to Evaluate AIDS (IeDEA, www.iedea.org) is a worldwide National Institute of Health sponsored collaboration of HIV treatment cohorts. This study included cohorts from Southern Africa, East Africa, and West Africa [21]. Data are collected on patients at the start of ART and at each follow-up visit. Clinic information includes demographic, clinical, and HIV regimen data. Before April 2010 (2007 in Zambia), if a patient experienced side effects or toxicities related to stavudine or zidovudine, and was not in need of second-line therapy, the recommendation was to substitute stavudine with either zidovudine, if no related anemia or neutropenia was present, or abacavir, and to substitute zidovudine with either stavudine or abacavir [22-28]. After April 2010 (2007 in Zambia), patients initiated onto stavudine or zidovudine now had tenofovir if no signs of renal insufficiency were detected, whereas those initiated onto tenofovir could substitute with stavudine, zidovudine, or abacavir [22-28].

All IeDEA sites obtained ethical approval from relevant local institutions before contributing anonymized patient data. Approval for analysis of de-identified data was granted by Boston University's Institutional Review Board. 


\section{Study design}

We performed a cohort analysis of data collected prospectively as part of routine care at clinics in the IeDEA multiregional collaboration. We included ARTnaïve, HIV-infected patients aged at least 16 years initiating first-line ART between 1 January 2005 and 31 December 2012, for all countries except Nigeria, where patients initiating ART between 1 January 2007 and 31 December 2012 were included as the roll out of ART started later. All patients had the potential for 24 months of follow-up. Prior to April 2010, national HIV treatment guidelines recommended the use of stavudine or zidovudine in first-line ART in all six included countries; thereafter, guidelines called for tenofovir or zidovudine to replace stavudine [22-26]. The only exception was in Zambia, which switched from stavudine or zidovudine to tenofovir in July $2007[27,28]$.

\section{Study variables}

All demographic (i.e. age, sex, clinic, and country) and clinical [i.e. year of ART initiation, $\mathrm{CD}^{+}$cell count, hemoglobin levels, weight, WHO stage, first-line NRTI (stavudine, zidovudine, or tenofovir), and non-nucleoside reverse transcriptase inhibitor (NNRTI) (nevirapine or efavirenz)] characteristics measured at ART initiation came from routinely collected clinic data. WHO staging was not available for East African countries.

The primary outcome variable was the proportion of patients who underwent a SDS in the first 24 months of ART. Follow-up time of 24 months was chosen as monitoring and time to development of toxicity/side effects differ between drugs. Laboratory monitoring for tenofovir and zidovudine is conducted early on after treatment initiation, whereas for stavudine, monitoring begins more often when the patient begins to develop clinical symptoms of toxicity (up to 24-48 months on ART [29]) diagnosed at a medical visit [2,3]. SDS was defined as substitution of the NRTI only within first-line ART. The reason for SDS was not available.

\section{Statistical analysis}

Patient characteristics at ART initiation were summarized with descriptive statistics and stratified by country. To look for trends in the use of stavudine in first-line ART and SDS over time, proportions of patients initiating stavudine or having a SDS in the first 24 months on ART were stratified by country and year of ART initiation, and plotted from 1 January 2005 to 31 December 2012, separately, with Nigeria being the exception, as data collection began in 2007. To test an additional hypothesis that tenofovir was being used among patients with contraindications to stavudine prior to guideline change, we looked at rates of SDS by NRTI over time.

Fine and Gray's [30] competing risks regression method was used to identify if the choice of NRTI in first-line ART was a predictor of SDS in the first 24 months on
ART, accounting for attrition as competing risks, and adjusted for age, sex, year of ART initiation, $\mathrm{CD}^{+}{ }^{+}$cell count, hemoglobin levels, WHO stage, and first-line NNRTI depending on country, with robust estimates at site level. We ran two models for each country. In both, we included all demographic and clinical characteristics at treatment initiation and year of ART initiation. The models differed as year of treatment initiation and NRTIs used were highly associated, and therefore each model used only one of the two. Follow-up time began at ART initiation and ended at the earliest of SDS; initiation of second-line ART; discontinuation of treatment; loss to follow-up (defined as not attending the clinic in the last 6 months); death; transfer; completion of 24-months of follow-up; or date of dataset closure (31 December 2014).

We assessed interaction between sex and NRTI, CD4 ${ }^{+}$ cell count and NRTI, hemoglobin levels and NRTI, $\mathrm{CD}^{+}$cell count and sex, WHO stage and sex, and hemoglobin levels and sex on the additive scale by calculating the risk due to interdependence $[\mathrm{R}(\mathrm{I})][31]$.

\section{Bayesian analysis}

As this is not the first study on the topic and we can draw stronger conclusions when incorporating those prior results into our current analysis, we conducted a Bayesian analysis which allows incorporation of the result of prior knowledge about the relationship between the exposure and the outcome into the estimation of parameters. Our approach to Bayesian analysis [32] is essentially a weighted average incorporating the prior distribution (previous literature) and our data. To do this, point estimates and corresponding 95\% CIs for priors were obtained from previous publications assessing predictors of SDS [12-20]. Ratio measures for each potential predictor of SDS (age, sex, clinic, $\mathrm{CD}^{+}$cell count, weight, hemoglobin levels, WHO stage, NRTI, and NNRTI used in first-line regimen) were extracted from the existing literature. We performed a meta-analysis using randomeffects models, due to heterogeneity in estimates, to first create weighted summary estimates for each individual predictor, separately, from the existing literature (referred to as the 'prior'). The same technique was used to calculate the summary estimates of each individual predictor, separately, from our data (referred to as the 'likelihood') and then combined the prior and likelihood estimates for each predictor, separately, to calculate a combined summary estimate (referred to as the 'posterior') and corresponding Bayesian credible intervals (CrIs).

\section{Sensitivity analysis}

As we may have had unmeasured confounding in our population, we conducted a multidimensional sensitivity analysis [33] by making assumptions about the strength of the effect of an unmeasured confounder on SDS and its prevalence in both patients on tenofovir or zidovudine (exposed) and those on stavudine (unexposed). We were 
interested in whether the confounder would overestimate the effect of exposure to tenofovir or zidovudine. We considered a confounder that would increase SDS and was more prevalent in patients on stavudine. We then back-calculated the relative risk we would have observed had we collected data on and adjusted for the purported confounder [33].

\section{Multiple imputation}

To account for missing data, we used multiple imputation by chained equations method using PROC MI in SAS [34] and assumed that the data were missing at random [35]. All models were fitted using 25 imputed datasets and estimated coefficients combined by averaging with the MIANALYZE procedure in SAS [36]. All clinical and demographic variables were included in the imputed models in addition to the outcome of SDS and indicator variables for death and loss to inform the missingness, but were not imputed. Appropriate standard errors were calculated using the within and between imputation standard errors of the estimates using Rubin's rules [35]. Since models based on imputed results did not differ from the models on the original data we displayed the results based on the original data.

\section{Results}

We included 377656 patients in the analysis (Table 1) $24 \%$ initiated a stavudine-based ART regimen, ranging from 25\% in Zambia and Uganda, to $60 \%$ in South Africa. Zambia contributed the largest number of patients $(n=205140)$ and Nigeria the smallest $(n=7434)$. Demographic and clinical characteristics were similar across countries. Patients were predominately female $(62.9 \%)$ with a median age of 35.2 years [interquartile range (IQR) 29.8-42.0] and a median time on treatment of 24.0 months (IQR 12.1-24.0), which did not differ by cohort. At ART initiation, patients had a median CD4 ${ }^{+}$ cell count of 155 cells/ $\mu$ l (IQR 74-241), with patients in South Africa having the lowest median $(130$ cells/ $\mu 1$, IQR 58-199) and Nigeria the highest (192 cells/ $\mu$ l, IQR 91-312).

When stratified by year, sex, weight, and age remained unchanged over time, whereas patients' cellular immunity at ART initiation improved in all countries. Additionally, the proportion of patients with tuberculosis and WHO III/IV stage declined over time in South Africa. Over $70 \%$ of patients in all countries remained alive and in care over 24 months on treatment. Overall attrition (combination of death and loss to follow-up) was $17.8 \%$ (95\% CI 17.7-17.9\%) and fairly consistent across countries, with Zambia having the lowest rate of attrition in the first 24 months on ART at 15.3\% (95\% CI $15.2-15.5 \%)$ and Nigeria the highest at $23.5 \%$ (95\% CI $22.5-24.4 \%)$.
Compliance with WHO guidelines: phasing out of stavudine in first-line antiretroviral therapy

All countries, with the exception of South Africa, where, in 2009, 95\% of patients still initiated stavudine, began phasing out stavudine prior to the WHO guidelines, making the change in 2010 (Fig. 1). Zambia, Kenya, and Uganda began replacing stavudine with zidovudine in first-line ART as early as 2007 (2005 in Zambia), potentially in parallel with the WHO's recommendation for lower-dose stavudine use $(30 \mathrm{mg}$ instead of $40 \mathrm{mg}$ [37]). Tenofovir was introduced after the WHO recommended its use in first-line therapy in 2010 (2007 in Zambia); as such, within 2 years of the change in 2012 , fewer than $10 \%$ of patients were being initiated on stavudine. Prior to 2010, in Cote d'Ivoire and Nigeria, stavudine and zidovudine were used interchangeably, whereas stavudine use decreased substantially, with less than $10 \%$ of patients initiating the drug after 2010 , when both countries decided on zidovudine as the NRTI of choice in first-line ART. Tenofovir was yet to be introduced in first-line ART in Cote d'Ivoire or Nigeria before 2012 due to cost [3].

\section{Decrease in single-drug substitutions associated with the phase out of stavudine}

Whereas the WHO policy change was accompanied by a clear shift away from stavudine to tenofovir, the impact on single-drug substitutions is less clear. Overall, SDS affected $8.8 \%$ (95\% CI $8.7-8.9 \%$ ) of patients in the first 24 months on ART, with $38 \%$ of SDS related to stavudine compared to 49 and $13 \%$ related to zidovudine and tenofovir, respectively. The decrease in SDS was associated with the phasing out of stavudine in firstline ART, decreasing from an overall rate of $11.3 \%$ (95\% CI $11.2-11.4 \%$ ) prior to 2010 , when $55 \%$ of patients were initiating stavudine, to $5.4 \%$ (95\% CI $5.3-5.5 \%$ ) after 2010 , when only $7.4 \%$ of patients initiated treatment with this drug. However, it is important to note that in all countries, SDS began roughly 2 years prior to the WHO guideline change in 2010 (Fig. 2). For all countries, competing risks regression models adjusted for year confirmed our results, showing a decrease in the hazards of SDS in accordance with the decrease in the use of stavudine (Supplementary Table 1, http://links.lww. com/QAD/A998).

\section{Single-drug substitutions stratified by initiating nucleoside reverse transcriptase inhibitor and substitution nucleoside reverse transcriptase inhibitor}

In addition to the variation observed in relation to the policy change, we also observed differences in rates of substitution by treatment regimen. Patients initiating stavudine $(13.9 \%$; 95\% CI 13.7-14.1\%) and zidovudine $(12.0 \%$; 95\% CI $11.8-12.2 \%)$ had higher rates of singledrug substitution compared to patients initiating tenofovir $(2.8 \%$; 95\% CI $2.7-2.9 \%)$. Also, whereas rates of substitution decreased over time for patients on tenofovir, 


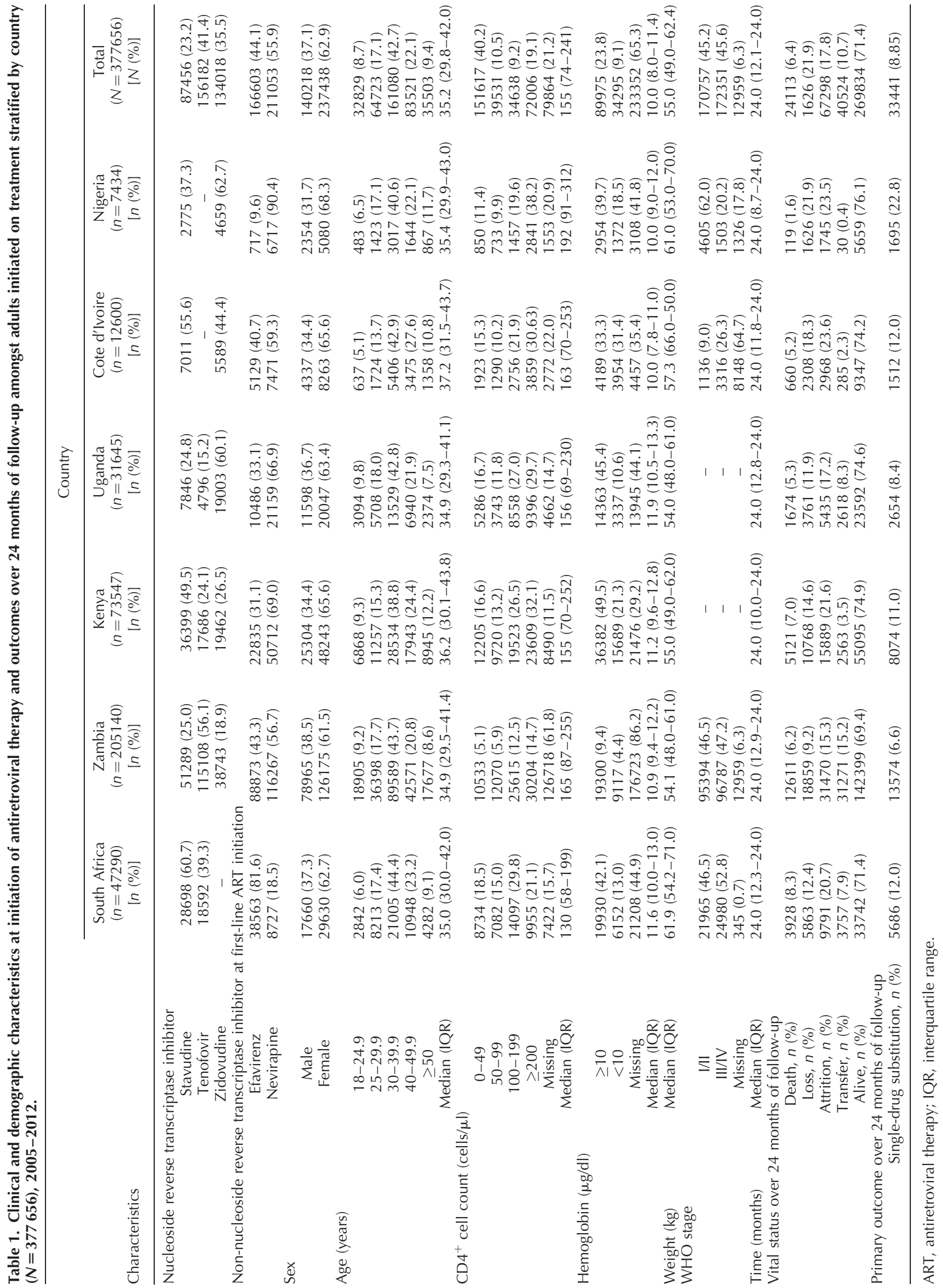




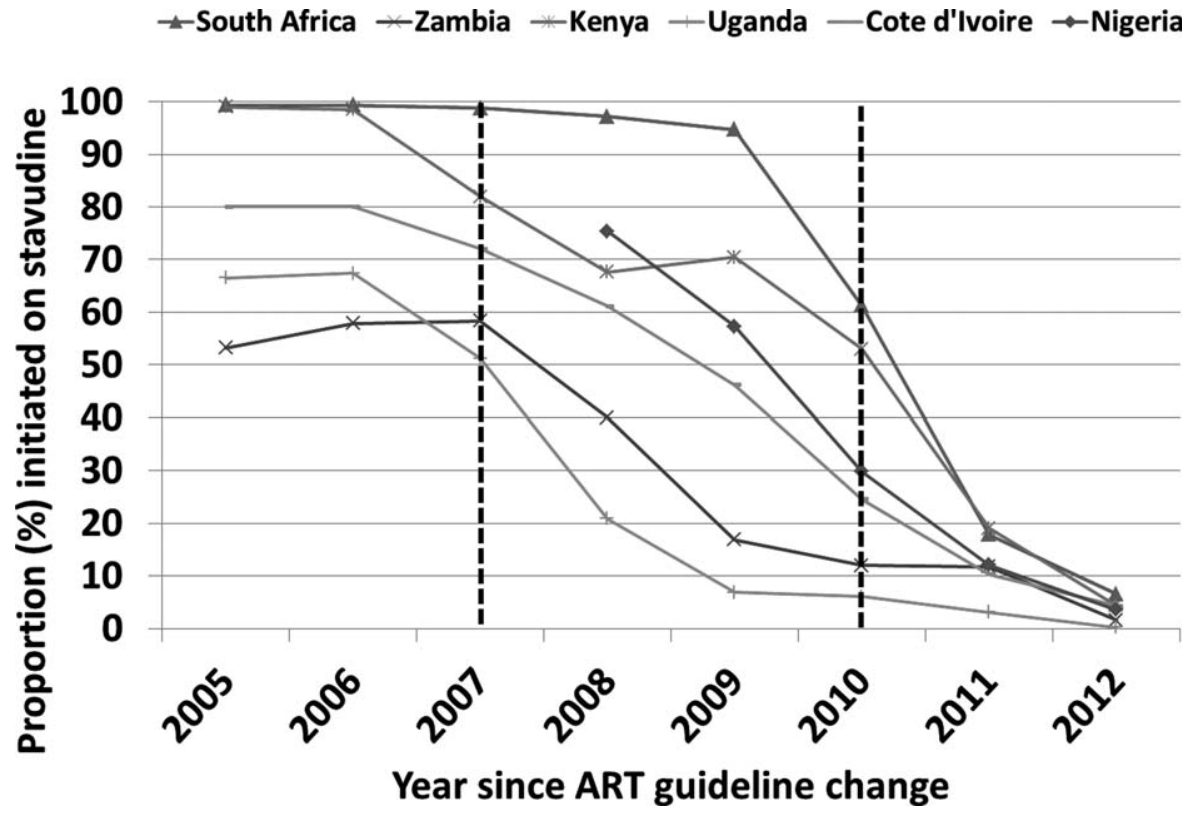

Fig. 1. Trends in the stavudine use in first-line ART for treatment-naive patients stratified by country and year since ART guidelines changed $(N=377$ 656). Dashed lines represent the change in national guidelines to introduce zidovudine or tenofovir into first-line ART (2007 for Zambia and 2010 for all other countries). ART, antiretroviral therapy.

they remained stable for zidovudine patients and increased for those on stavudine (Supplementary Fig. 1, http://links.lww.com/QAD/A998). All countries followed the national ART guidelines outlining the antiretrovirals eligible for substitution. These included tenofovir, stavudine, zidovudine, or abacavir depending on NRTIs included in the first-line regimen with the occasional use of didanosine as an alternative(Supplementary Fig. 2, http://links.lww.com/QAD/A998). Zidovudine was the most common SDS used for both tenofovir (50\%; 95\% CI 48.6-51.6\%) and stavudine (67.9\%; 95\% CI 67.2-68.6\%), whereas stavudine was the

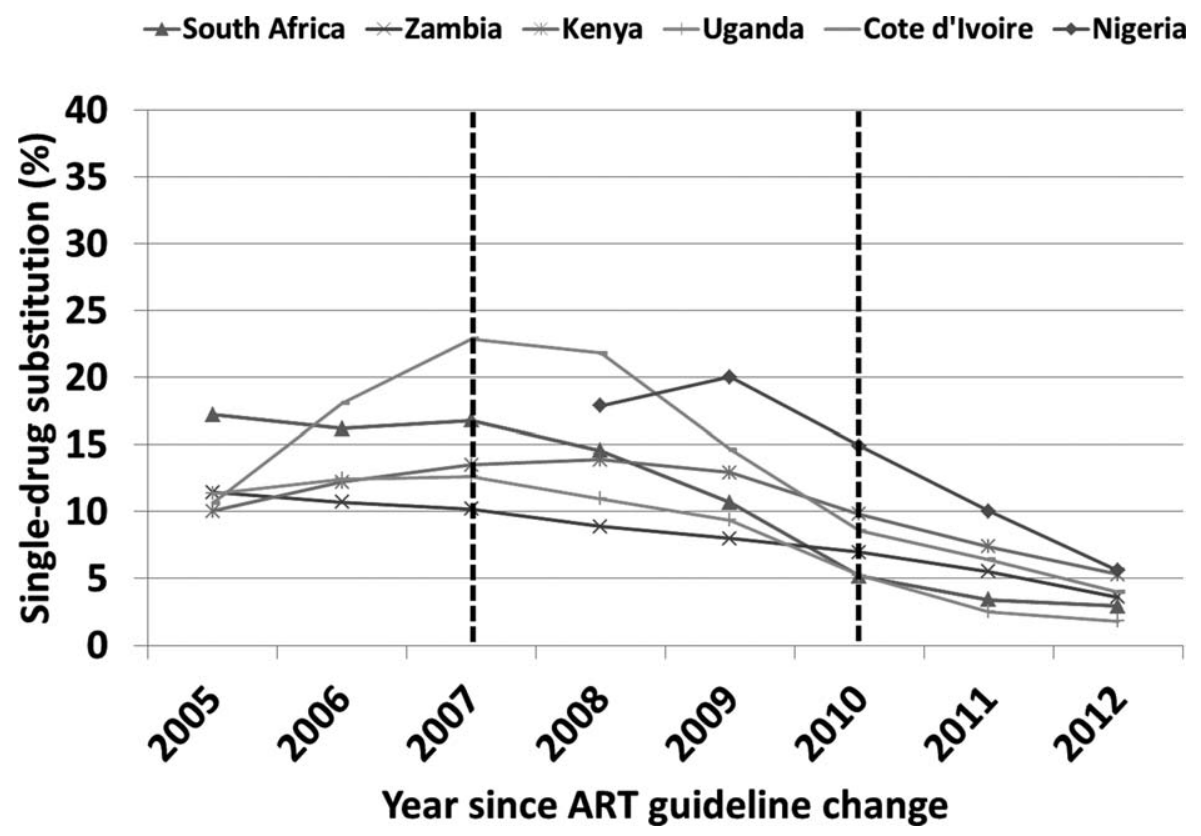

Fig. 2. Proportion of single-drug substitutions over the first 24 months on ART stratified by year since ART guidelines changed for all countries $(N=377$ 656). Dashed lines represents the change in national guidelines to introduce zidovudine or tenofovir into first-line ART (2007 for Zambia and 2010 for all other countries). ART, antiretroviral therapy. 
SDS for a zidovudine-based first-line regimen $(65.1 \%$; 95\% CI 64.2-66.0\%).

Consistent with known toxicity patterns, SDS occurred earlier for patients on zidovudine (median 8.1 months after start of treatment; IQR 2.3-17.0) and tenofovir (median 10.2 months after start of treatment; IQR 3.618.1) compared to stavudine(median 14.2 months; IQR 7.3-19.6) after start of treatment.

\section{Changing the nucleoside reverse transcriptase inhibitor used in first-line antiretroviral therapy could explain the temporal trends in single-drug substitutions}

Adjusted competing risks regression models evaluating the association between choice of NRTI used in first-line ART and SDS helped us to confirm that the decrease in rates of SDS was associated with the phasing out of stavudine for tenofovir or zidovudine in first-line ART. Adjusted models showed that patients initiating tenofovir in Southern and East Africa were 20-95\% less likely to undergo a substitution than patients initiating stavudine (Table 2). Posterior Bayesian estimates using an informative prior showed close to an $80 \%$ reduction in the risk of SDS (posterior risk ratio $0.21,95 \% \mathrm{CrI} 0.20-0.22$ ) when comparing tenofovir to stavudine (Table 3). With the exception of Zambia, where we saw an increase in the hazards of SDS when comparing zidovudine to stavudine (hazard ratio 2.59, 95\% CI 2.3-3.0) and Kenya, where we saw no association (HR 1.13, 95\% CI 0.99-1.28) (Table 2 ), patients on zidovudine compared to stavudine in Uganda, Cote d'Ivoire, and Nigeria had a 75-85\% decrease in the hazards of SDS when compared to stavudine. Posterior Bayesian estimates using an informative prior showed close to a 70\% reduction in the risk of SDS (posterior risk ratio $0.31,95 \%$ CrI $0.30-0.33$ ) (Table 3).

Our results also suggest that females compared to males have a $50 \%$ increase in the risk of SDS (posterior risk ratio 1.48, 95\% CrI 1.43-1.52) (Tables 2 and 3). Since we believed changes in the trends of substitutions for sex could vary by NRTI used in first-line ART, we calculated the risk due to interdependence $[\mathrm{R}(\mathrm{I})]$. With the exception of Zambia and Kenya, where the R(I) was essentially 0 , the other four countries showed a positive interdependence (Uganda 2\%, Cote d'Ivoire 4\%, South Africa 5\%, and Nigeria 6\%). In other words, in Nigeria, for example, $6 \%$ of SDS in women on stavudine is related to the dual action of female sex and stavudine. The risk of SDS in the doubly exposed (females exposed to stavudine) was $41.9 \%$ versus the risk of $6.0 \%$ in the doubly unexposed (males unexposed to stavudine). We did not see any signs of interaction when assessing all other biological relationships using the $\mathrm{R}(\mathrm{I})$.

We saw inconsistent results when comparing nevirapine to efavirenz. In Zambia, Kenya, Uganda, and Nigeria, patients on nevirapine had a $10-70 \%$ increase in the risk of SDS compared to those on efavirenz, whereas patients in South Africa and Cote D'Ivoire had a decrease in SDS of 20 and $40 \%$, respectively. We also saw a decrease in the risk of SDS by $10-40 \%$ in all countries amongst patients with $\mathrm{CD}^{+}$cell count below 100 cells/ $\mu$ l compared to at least 100 cells $/ \mu 1$.

\section{Bias analysis}

Bias analyses simulating a confounder that would overestimate the effect of tenofovir or zidovudine versus stavudine showed that in order for adjustment for an unmeasured confounder to bring our results close to null, the confounder would have to be present in 5\% in those exposed to tenofovir or zidovudine, extremely common among patients exposed to stavudine (40\%), and be a very strong predictor of SDS (risk ratio >11.3), highly unlikely (Supplementary Table 2, http://links.lww.com/QAD/ A998).

\section{Discussion}

In the largest study to date, across multiple countries in the African continent, we show steady decrease in SDS corresponding to the phasing out of stavudine, in accordance with the WHO guidelines, from an overall rate of $11 \%$ prior to 2010 , when $55 \%$ of patients were initiating stavudine, to $5 \%$ after 2010 , when only $7 \%$ of patients initiated treatment with this drug. Using Bayesian methods [32], although there was high heterogeneity between studies for the majority of estimates, we were able to estimate an $80 \%$ decrease in the risk of SDS when comparing tenofovir to stavudine, and $70 \%$ decrease when comparing stavudine to zidovudine, further highlighting the better safety profile associated with tenofovir and zidovudine compared to stavudine. Our results also showed a decrease in the rates of SDS from 2005 to 2012 for patients on tenofovir, providing evidence to support the notion that patients in earlier years were being initiated on tenofovir due to contraindications to stavudine or zidovudine, and were therefore at a higher risk of toxicity/side effects.

Time to substitution varied depending on the NRTI used in first-line ART, with zidovudine and tenofovir occurring early after treatment initiation and at a higher rate later on in follow-up amongst patients on stavudine. This is consistent with previous studies conducted in subSaharan Africa [12-20]; however, it is important to note that time to substitution is partly a function of the frequency of monitoring, which differs for each NRTI. Laboratory monitoring for tenofovir and zidovudine is often conducted early on after treatment initiation, whereas for stavudine, monitoring begins more often when the patient begins to develop clinical symptoms of toxicity diagnosed at a medical visit [2,3]. Additionally, although national guidelines for substitution of the NRTI 


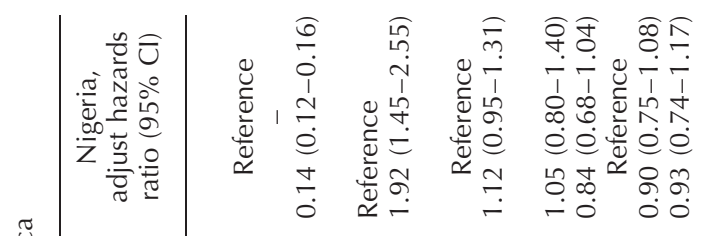

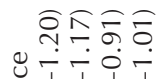

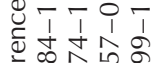

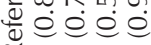

$\propto$ कार 8

产

茛

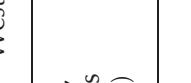

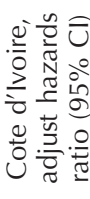

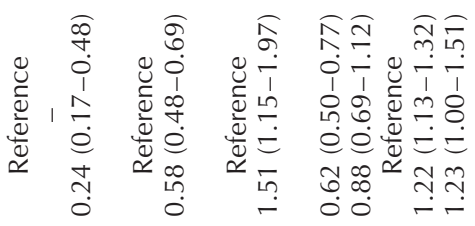

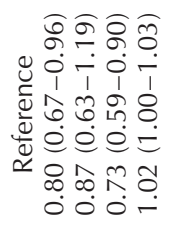

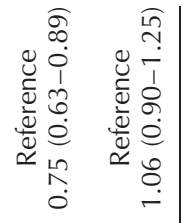

.

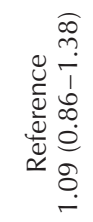

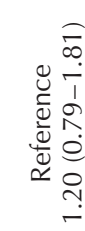

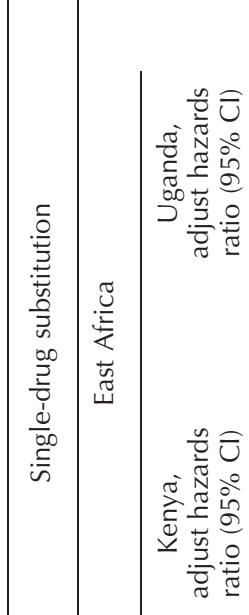

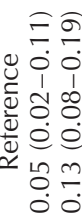

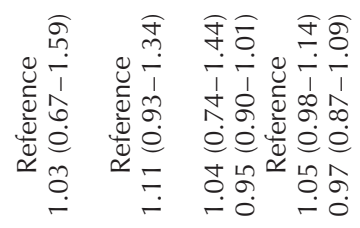

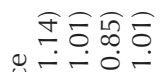

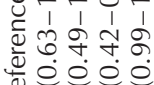

๘ंडe

$\simeq$ 는요용

०00-

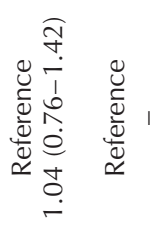

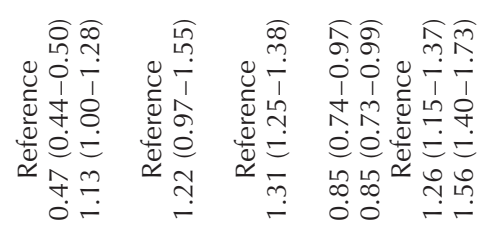

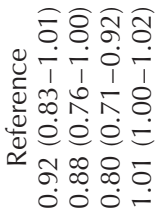

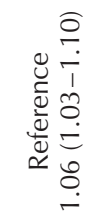

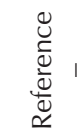

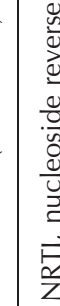

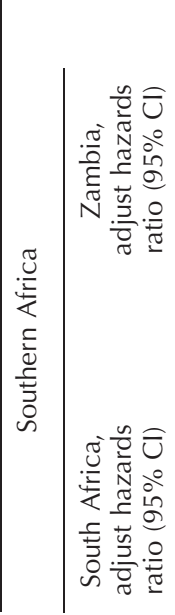

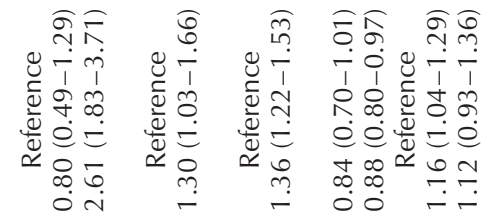

ิㅡํํำ

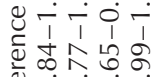

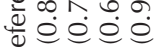

$\propto$ न $\infty$ o

000
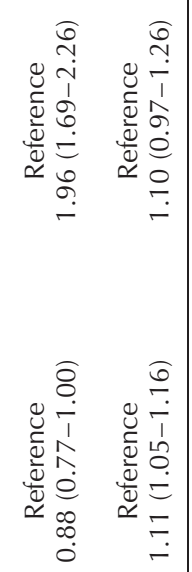

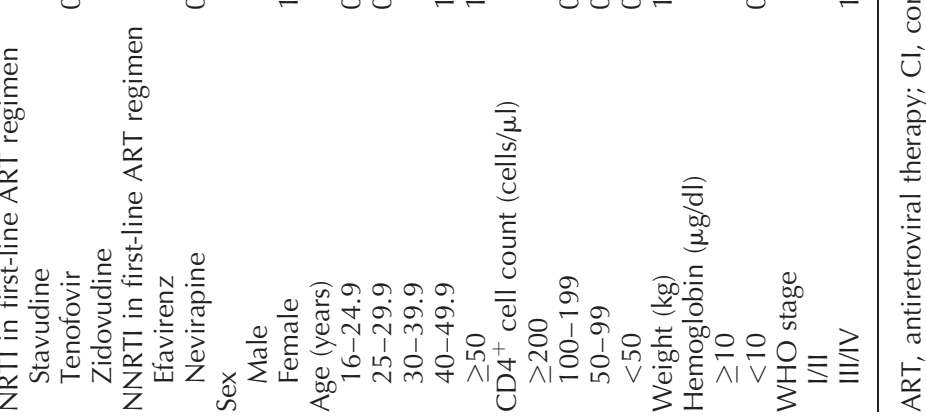




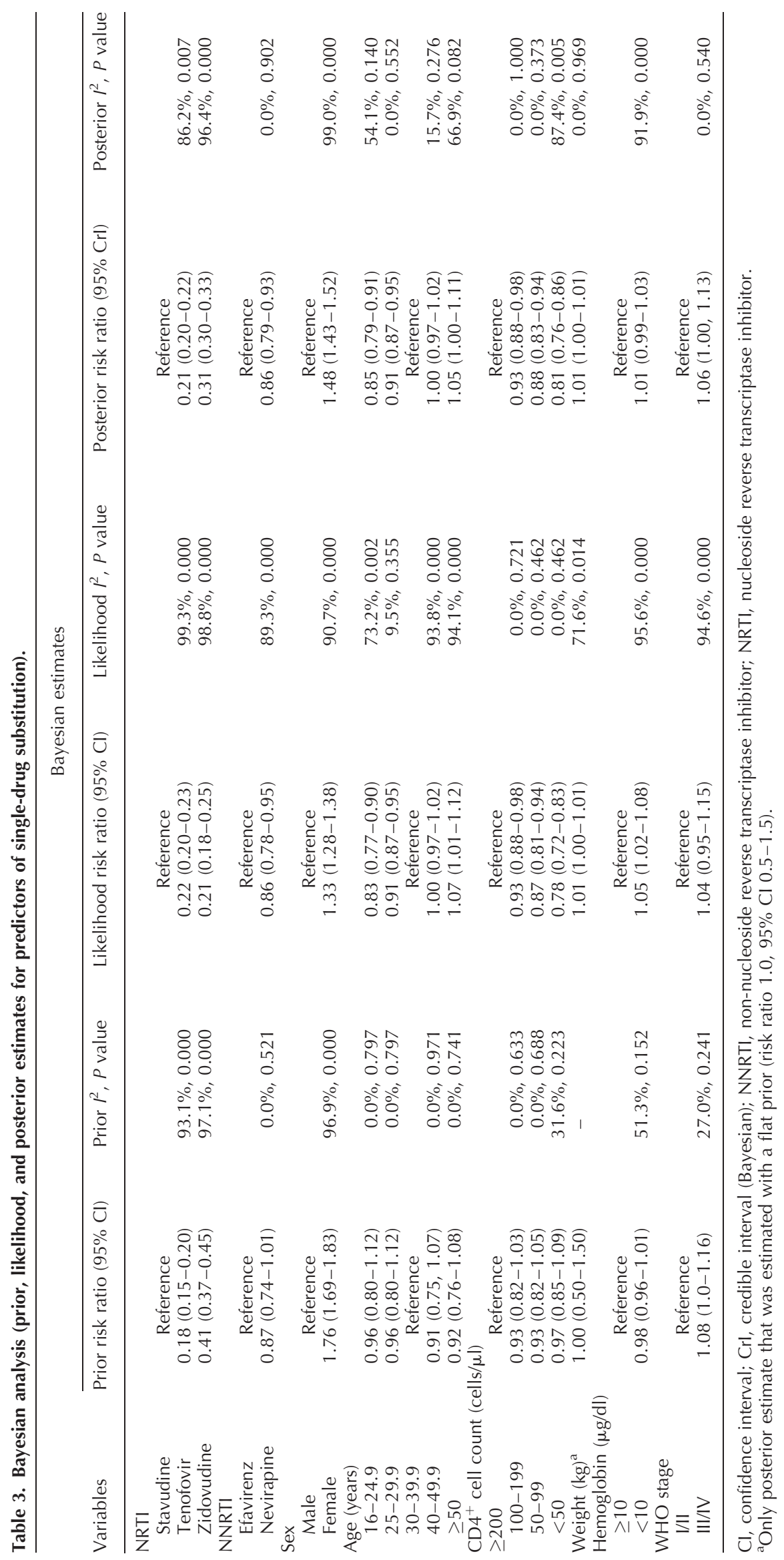


and NNRTI within first-line ART were the same, the difference in estimates when comparing zidovudine to stavudine, and also nevirapine to efavirenz could be due to variation in monitoring practices or the availability of NRTIs for substitution in each country.

Females, compared to males, had a 50\% increase in the risk of SDS in our study, consistent with previous research [12,14-19]. By assessing effect measure modification on the additive scale, we also showed that depending on country, $2-6 \%$ of SDS in women on stavudine were related to the dual action of female sex and stavudine. Although we could not confirm the reason for substitution in our study, previous research has reported women on stavudine are at a higher risk of toxicity/side effects than men $[29,38,39]$, and that differences in risk of toxicity observed between sexes could be related to differences in susceptibility or to a higher level of adherence to therapy achieved by women [29]. In addition to the size of our study population, we were the first to evaluate trends in SDS over almost a decade of treatment in public sector since the roll out of ART in 2004 in six countries in sub-Saharan Africa. However, our findings should be considered in light of the study limitations. First, this study represents patients from urban areas. Although some clinics are run out of tertiary hospitals, the majority operate at the primary care level, are led by nurses or clinical officers rather than physicians, and are part of the public healthcare system of the country, and may, therefore, not be generalizable to other clinics. Second, there was variability in the estimates of the association between NRTI used in first-line ART and SDS by country. When comparing tenofovir to stavudine, we saw a $20-95 \%$ decrease in the hazards of SDS, and when comparing zidovudine to stavudine, we saw a $85 \%$ reduction to a $200 \%$ increase in the hazards of SDS. Although national guidelines for substitution of the NRTI within first-line ART were the same in all six countries, the difference could be due to variation in monitoring practices at the level of the clinic or clinician or the availability of alternative NRTIs used for substitution. Third, due to the lack of documentation of reasons for SDS for almost 95\% of events amongst patients with the event, we are likely underestimating the frequency and type of side effects due to less-than-perfect surveillance. There is a chance that SDS in our study were driven by the policy change and not by side effects/ toxicity of stavudine. When we conducted this study in our pediatric population, we did see a substantial spike in SDS around the time of the guideline change as clinicians were substituting stavudine, regardless of the fact that the patient was tolerating stavudine well, with zidovudine or tenofovir. If substitutions were being driven by the guidelines in adults, we believe we would see a similar increase, which is not present in these data. Fourth, patients with lower $\mathrm{CD} 4{ }^{+}$cell count $(<100$ cells $/ \mu \mathrm{l})$ had upwards of $40 \%$ decrease in the risk of SDS over the follow-up period in some countries. As we do see higher rates of attrition in patients with a lower $\mathrm{CD} 4^{+}$cell count $(<100$ cells $/ \mu l$ to $23.6 \%$ vs. $\geq 100$ cells $/ \mu l$ to $14.2 \%)$, the decreased risk amongst patients with poorer immune status could be caused by survivor bias. Fifth, in order to strengthen the argument for further reduction in the cost in tenofovir and zidovudine for use in LMICs, we recognize that it would be important to know the proportion of individuals who are symptomatic with toxicity related to stavudine-based therapy and did not experience a SDS. Unfortunately, conditions are poorly captured in the database, preventing us from accurately assessing symptoms among patients who did not have a SDS. However, Fig. 1 shows that the policy change switching to tenofovir was the reason for the decline in SDS in South Africa, as the country went from almost $100 \%$ of patients initiating stavudine prior to the 2010 guidelines changing to less than $10 \%$, which we believe allowed us to evaluate what a change in national policy to included tenofovir in first-line ART might do in other countries as they make the change. Sixth, it is possible that our populations differed with respect to some unmeasured confounders, as data on WHO staging were not available for Kenya and Uganda. We may therefore have residual confounding in our estimates from those countries. However, our bias analysis suggests such an unmeasured confounder would be extremely unlikely in our cohort. Finally, multiple imputation helps make it possible to handle missing data routinely and improve the validity of research. However, deviations from the assumptions needed [34] could have led to unpredictable biases in our parameter estimates.

\section{Conclusion}

The decline in SDS in the first 24 months on treatment is associated with phasing out of stavudine for zidovudine or tenofovir in first-line ART in the countries included in our study. When calculating the number needed to treat, we found that to prevent one additional SDS event, six patients would need to be treated over 24 months, which would be considered beneficial, supporting further efforts to decrease the cost of tenofovir and zidovudine for use in LMICs.

\section{Acknowledgments}

Funding was provided by USAID under the terms of INROADS USAID-674-A-12-00029 and the National Institutes of Allergy and Infectious Diseases (NIAID) the IeDEA initiative Award Number 2U01AI069924, IeDEA-Southern Africa Award Number U01AI069924-10, IeDEA-East Africa Award Number U01AI0699-11, IeDEA-West Africa Award Number U01AI069919-10. This study is made possible by the generous support of the American people through the United States Agency for International Development (USAID) and the National Institutes of Health. The 
contents are the responsibility of the authors and do not necessarily reflect the views of USAID, NIH or the United States government.

\section{Conflicts of interest}

There are no conflicts of interest.

\section{References}

1. Global Health Sector Response to HIV, 2000-2015. Focus on innovations in Africa. http://apps.who.int/iris/bitstream/10665/ 198065/1/9789241509824_eng.pdf [Accessed 1 May 2016].

2. World Health Organization. Scaling up antiretroviral therapy in resource-limited settings: treatment guidelines for public health approach. 2003 Revision. Geneva, Switzerland: WHO; 2004.

3. World Health Organization. Antiretroviral therapy for HIV infection in adults and adolescents: recommendations for a public health approach. 2010 Revision. Geneva, Switzerland: WHO; 2010.

4. Gallant JE, Staszewski S, Pozniak AL, Dejesus E, Suleiman JMAH, Miller MD, et al. Efficacy and safety of tenofovir DF vs. stavudine in combination therapy in antiretroviral-naive patients: a 3-year randomized trial. / Am Med Assoc 2004; 292:191-201.

5. Domingos H, Cunha RVD, Paniago AMM, Martins DM, Elkhoury EB, Souza ASD. Metabolic effects associated to the highly active antiretroviral therapy (HAART) in AIDS patients. Braz J Infect Dis 2009; 13:130-136.

6. van Griensven J, Zachariah R, Rasschaert F, Mugabo J, Atté EF, Reid T. Stavudine- and nevirapine-related drug toxicity while on generic fixed-dose antiretroviral treatment: incidence, timing and risk factors in a three-year cohort in Kigali, Rwanda. Trans R Soc Trop Med Hyg 2010; 104:148-153.

7. Cournil A, Coudray M, Kouanfack C, Essomba CN, Tonfack CAD, Biwolé-Sida $M$, et al. Reduced dose of stavudine and lipoatrophy in HIV-infected patients in Cameroon. Antivir Ther 2010; 15:1039-1043.

8. Global HIV/AIDS response. Epidemic update and health sector progress towards Universal Access. Progress Report 2011. Geneva, Switzerland: WHO/UNAIDS; 2010.

9. Menezes CN, Crowther NJ, Duarte R, Van Amsterdam D, Evans $\mathrm{D}$, Dickens $\mathrm{C}$, et al. A randomized clinical trial comparing metabolic parameters after 48 weeks of standard- and low-dose stavudine therapy and tenofovir disoproxil fumarate therapy in HIV-infected South African patients. HIV Med 2014; 15:3-12.

10. Arribas JR, Pozniak AL, Gallant JE, Dejesus E, Gazzard B, Campo RE, et al. Tenofovir disoproxil fumarate, emtricitabine, and efavirenz compared with zidovudine/lamivudine and efavirenz in treatment-naive patients: 144-week analysis. J Acquir Immune Defic Syndr 2008; 47:74-78.

11. Haubrich RH, Riddler SA, DiRienzo AG, Komarow L, Powderly WG, Klingman K, et al. Metabolic outcomes in a randomized trial of nucleoside, non-nucleoside and protease inhibitor-sparing regimens for initial HIV treatment. AIDS 2009; 23:1109-1118.

12. Velen K, Lewis JJ, Charalambous S, Grant AD, Churchyard GJ, Hoffmann CJ. Comparison of tenofovir, zidovudine, or stavudine as part of first-line antiretroviral therapy in a resourcelimited-setting: a cohort study. PLoS One 2013; 8:e64459.

13. Bygrave $\mathrm{H}$, Ford $\mathrm{N}$, van Cutsem $\mathrm{G}$, Hilderbrand $\mathrm{K}$, Jouquet $\mathrm{G}$, Goemaere $\mathrm{E}$, et al. Implementing a tenofovir-based first-line regimen in rural Lesotho: clinical outcomes and toxicities after two years. J Acquir Immune Defic Syndr 2011; 56:e75-e78.

14. Chi BH, Mwango A, Giganti M, Mulenga LB, TambatambaChapula B, Reid SE, et al. Early clinical and programmatic outcomes with tenofovir-based antiretroviral therapy in Zambia. I Acquir Immune Defic Syndr 2010; 54:63-70.

15. Brennan AT, Maskew M, Ive P, Shearer K, Long L, Sanne I, et al. Increases in regimen durability associated with the introduction of tenofovir at a large public-sector clinic in Johannesburg, South Africa. J Int AIDS SOC 2013; 16:18794.

16. Njuguna C, Orrell C, Kaplan R, Bekker LG, Wood R, Lawn SD. Rates of switching antiretroviral drugs in a primary care service in South Africa before and after introduction of tenofovir. PLOS One 2013; 8:e63596.
17. Inzaule S, Otieno J, Kalyango J, Nafisa L, Kabugo C, Nalusiba J, et al. Incidence and predictors of first line antiretroviral regimen modification in western Kenya. PLoS One 2014; 9:e93106.

18. Labhardt ND, Sello M, Lejone T, Ehmer J, Mokhantso M, Lynen $\mathrm{L}$, et al. Adoption of new HIV treatment guidelines and drug substitutions within first-line as a measure of quality of care in rural Lesotho: health centers and hospitals compared. Trop Med Int Health 2012; 17:1245-1254.

19. Boulle A, Orrel C, Kaplan R, Van Cutsem G, McNally M, Hilderbrand $\mathrm{K}$, et al. Substitutions due to antiretroviral toxicity or contraindication in the first 3 years of antiretroviral therapy in a large South African cohort. International Epidemiological Databases to Evaluate Aids in Southern Africa Collaboration. Antivir Ther 2007; 12:753-760.

20. Takuva S, Louwagie G, Zuma K, Okello V. Durability of first line antiretroviral therapy: reasons and predictive factors for modifications in a Swaziland cohort. J Antivir Antiretrovir 2012; 4:014-020.

21. Egger M, Ekouevi DK, Williams $\mathrm{C}$, Lyamuya RE, Mukumbi $\mathrm{H}$, Braitstein $\mathrm{P}$, et al. Cohort profile: the international epidemiological databases to evaluate AIDS (IeDEA) in sub-Saharan Africa. Int / Epidemiol 2012; 41:1256-1264.

22. National Department of Health, Republic of South Africa. The South African Antiretroviral Treatment Guidelines. 2010. http:// www.doh.gov.za/docs/factsheets/guidelines/art.pdf [Accessed 1 May 2016].

23. Ministry of Health, Government of Kenya. Guidelines for antiretroviral drug therapy in Kenya, 4th ed. 2011. http://healthser vices.uonbi.ac.ke/sites/default/files/centraladmin/healthservices/ Kenya $\% 20$ Treatment $\% 20$ Guidelines $\% 202011$.pdf [Accessed 1 May 2016]

24. Ministry of Health, Government of Uganda (2009): National Antiretroviral treatment guidelines for Adults, adolescents and children. MOH 2009, 3rd ed. http://apps.who.int/medicinedocs/ documents/s19249en/s19249en.pdf [Accessed 1 May 2016].

25. Ministère de la Santé et de l'Hygiène Publique de la République de Côte d'Ivoire, Programme National de Prise en Charge des Personnes Vivant avec le VIH. Atelier technique de révision des Stratégies thérapeutiques antirétrovirales en Côte d'Ivoire; Bassam. 12 - 15 février 2008; Rapport final.

26. Federal Ministry of Health. Department of Public Health Nigeria (2010). National guidelines for HIV and AIDS treatment and care in adolescents and adults. http://www.who.int/hiv/pub/ guidelines/nigeria_art.pdf [Accessed 1 May 2016].

27. Ministry of Health, Republic of Zambia. Adult and Adolescent Antiretroviral Therapy Protocols; 2010. http://www.who.int/ hiv/pub/guidelines/zambia_art.pdf [Accessed 1 May 2016].

28. Mwinga A. Inclusion of a tenofovir-based first-line regimen in Zambia: a bold step forward? Paper presented at: 2007 HIV/ AIDS Implementer's Meeting; Kigali, Rwanda; 2007.

29. Pujades-Rodríguez M, Dantony E, Pinoges L, Ecochard R, Etard JF, Carrillo-Casas E, et al., For the AIDS Working Group of Médecins Sans Frontières. Toxicity associated with stavudine dose reduction from 40 to $30 \mathrm{mg}$ in first-line antiretroviraltherapy. PLoS One 2011; 6:e28112.

30. Fine JP, Gray RJ. A proportional hazards model for the sub distribution of a competing risk. I Am Stat Assoc 1999; 94:496-509.

31. Rothman KJ. Interactions between causes. Mod Epidemiol 1986:311-326.

32. MacLehose RF, Ghassan BH. Applications of Bayesian methods to epidemiologic research. Curr Epidemiol Rep 2014:103-1091.3.

33. Lash TL, Fox MP, Fink AK. Applying quantitative bias analysis to epidemiologic Data.Springer; 2009.

34. The MI Procedure. http://support.sas.com/rnd/app/papers/ miv802.pdf [Accessed 1 May 2016].

35. Rubin DB. Multiple imputation for nonresponse in surveys. New York: John Wiley \& Sons; 1987.

36. The MIANALYZE Procedure. http://support.sas.com/rnd/app/ papers/mianalyzev802.pdf [Accessed 1 May 2016].

37. World Health Organization. Addendum to 2006 WHO guidelines on antiretroviral therapy for HIV infection in adults and adolescents. Geneva, Switzerland: WHO; 2007.

38. Emery J, Pick N, Mills EJ, Cooper CL. Gender differences in clinical, immunological, and virological outcomes in highly active antiretroviral-treated HIV-HCV coinfected patients. $\mathrm{Pa}$ tient Prefer Adherence 2010; 4:97-103.

39. Kempf MC, Pisu M, Dumcheva A, Westfall AO, Kilby JM, Saag MS. Gender differences in discontinuation of antiretroviral treatment regimens. I Acquir Immune Defic Syndr 2009; 52:336-341. 